\title{
Las relaciones de género en las políticas locales y en el desarrollo económico del Pirineo Catalán*
}

\author{
Gender relations in local politics and economic development in the \\ Catalan Pyrenees
}

\author{
Antoni F. Tulla \\ Universidad Autónoma de Barcelona \\ antoni.tulla@uab.es \\ Antonia Casellas \\ Universidad Autónoma de Barcelona \\ antonia.casellas@uab.cat \\ Marta Pallares-Blanch \\ Universidad Autónoma de Barcelona \\ marta.pallares@gmail.com

\begin{abstract}
Ana Vera
Universidad Autónoma de Barcelona

ana.vera@uab.cat
\end{abstract}

\begin{abstract}
Resumo
El presente artículo explora la contribución de las mujeres en las estrategias de desarrollo local en un área de montaña. El estudio analiza roles políticos y económicos desde una perspectiva de género en la Cataluña rural. Se considera la sorprendentemente baja participación en la política local de las mujeres en Cataluña en comparación con el conjunto de España, al igual que la medida en que las decisiones de las mujeres difieren de las de los hombres con respecto a los enfoques empresariales para el desarrollo económico local. El documento también analiza la participación de las mujeres en la toma de decisiones, investigando si tienen la capacidad de involucrar a actores locales innovadores en la formulación de políticas. Se presentan las características económicas del Pirineo Catalán. La investigación aplica metodologías cualitativas y cuantitativas, incluyendo entrevistas en profundidad e información elaborada a través de bases de datos.
\end{abstract}

Palabras clave: administración local; desarrollo económico local; metodología cualitativa; Pirineo Catalán; relaciones de género en política.

\begin{abstract}
The present paper explores women's contribution to local development strategies in a mountainous area. The study analyses gender-based political and economic roles in rural Catalonia. It is considered both Catalonia's surprisingly low rate of women's political participation, when compared to Spain as a whole, and the extent to which women's decisions can be significantly different than men's ones with respect to entrepreneurial approaches to local economic development. The paper further analyses women's participation in decision-making, investigating whether they have the capacity to involve innovative stakeholders in local policy-making. The economic characteristics of the Pyrenees region are addressed. The research applies both qualitative and quantitative methodology, including in-depth interviews and information gathered from databases.
\end{abstract}

Keywords: local administration; local economic development; qualitative methodology; Catalan Pyrenees, gender relations in politics. 
La participación de las mujeres en los cargos políticos elegidos, a partir de la recuperación de la democracia en España (1976), se ha ido ampliando desde una situación testimonial $(4,57 \%$ de diputadas al congreso en 1982-86) hasta porcentajes cercanos al 40\% (36\% 2011-2015) (Instituto de la Mujer, 2011). Esta evolución ha sido desigual según el ámbito territorial (municipal, autonómico o estatal) y con el tipo de área (urbanas, rurales, centrales, periféricas, etc.) en que se lleva a cabo. En nuestra investigación hemos analizado los datos de las elecciones municipales en el Alto Pirineo Catalán (APC), un área montañosa y periférica de Cataluña. En estudios preliminares (CASELLAS et al, 2009, p. 16-20) se puede constatar que el porcentaje de mujeres regidoras es menor en Cataluña que en el resto de España y, así mismo, es aún menor en los municipios del APC. En general, se puede afirmar (a) que, proporcionalmente, hay menos alcaldesas que regidoras en todos los municipios, (b) que hay un mayor nombre de regidoras en los municipios urbanos, pero (c) hay un número relativo mayor de alcaldesas en los pequeños municipios, tal como puede deducirse en la Tabla 1 o en la Tabla 5 cuando estudiemos el APC.

Si nos fijamos en la evolución general del Estado español, observamos que se ha evolucionado de un $2 \%$ de alcaldesas en el año 1983 hasta casi un 15\% en el 2007. El porcentaje de regidoras era sólo el 6,5\% en 1995 llegando a un $30,5 \%$ en el 2007, siendo más del doble que el porcentaje de alcaldesas. En regiones urbanas como el País Vasco (19,9\%), Madrid (19,6\%) o Valencia $(17,5 \%)$ el porcentaje de alcaldesas es superior al de la media española con la excepción de Cataluña $(12,7 \%)$. En cambio, en dichas regiones urbanas el porcentaje de regidoras es ligeramente inferior a la media mientras que en las áreas más rurales como Canarias (38,3\%), Andalucía (37,8\%), Murcia (38,9\%), La Rioja (35\%) o Extremadura (35\%) es muy superior a la media. Se debe citar que la Ley Orgánica 3/2007 para la Igualdad Efectiva de Mujeres y Hombres ha promovido un número igual de hombres y mujeres en las listas de municipios de más de 5.000 h. en las elecciones realizadas desde el año 2007.

En las áreas de montaña de Cataluña, aún predomina el patriarcado en las relaciones económicas y sociales (TULLA, 1991; TULLA; 1994). Las decisiones en la esfera pública son tomadas por los hombres $y$, en este sentido, la representación política por parte de las mujeres ha sido ocasional hasta los últimos años. Las distintas formas y grados de patriarcado pueden variar de un país a otro y de acuerdo con determinadas circunstancias económicas y sociales (DUNCAN, 1996). En las áreas rurales del APC, el predominio explícito e implícito de la figura jurídica del hereu ${ }^{1}$, que ya ha perdido importancia en las áreas urbanas, acentúa la posición de poder masculino. Se entiende que las decisiones económicas y políticas en el ámbito público corresponden al hombre, remitiendo a la mujer a las decisiones que se toman en el ámbito privado. Así, éstas pueden tener empleos fuera del ámbito familiar pero en las áreas rurales siempre se considerará que deben priorizar las responsabilidades familiares sobre las públicas (TULLA, 1991). Ello tiene una clara repercusión en el acceso de la mujer a los cargos de representación política en la administración local. El objetivo de nuestra investigación es demostrar esta situación marginal de la mujer que implica que no debe acceder a cargos políticos públicos, y si lo hace, es juzgada con más rigor que los hombres (BAYLINA ; BOCK, 2004).

\section{Metodología}

En esta investigación nos hemos planteado utilizar tres enfoques metodológicos. Primero, la revisión bibliográfica que se centra en la participación de la mujer en los cargos electos y de gobierno en las instituciones políticas de España, en general, y de Cataluña en particular, y al mismo tiempo la participación de la mujer en las decisiones económicas, especialmente en el desarrollo local y el desarrollo rural integrado. Segundo, se ha analizado la información cuantitativa recogida (datos secundarios) referente a los cargos electos individuales en la administración local del APC y del conjunto de Cataluña y España (CORCOY, 2008). El análisis cuantitativo considera cinco momentos históricos: el período legislativo (PL) 1979-83 por ser el más próximo a la dictadura, el PL 1991-95, como intermedio, el PL 2003-07 por ser un cambio de etapa en el que muchos cargos no repiten, y el PL 2007-11, que es el más actual. El PL 2011-15 se utilizará para comentarios puntuales. Tercero, el análisis cualitativo comprende la realización de 30 entrevistas en profundidad a una selección de mujeres y hombres que han ocupado, o están ocupando, cargos electos en la administración local, para profundizar en la situación real de la mujer en la administración local, comparándola con la de los hombres. Se incluyen también entrevistas a especialistas de la gestión económica y del desarrollo local del APC (Tabla 2). 
Las relaciones de género en las políticas locales y en el desarrollo económico del Pirineo Catalán

\begin{tabular}{|c|c|c|c|c|c|c|c|c|c|c|c|c|c|c|c|c|}
\hline \multirow{3}{*}{$\begin{array}{c}\text { Comunidades } \\
\text { Autónomas (regiones) }\end{array}$} & \multirow{2}{*}{\multicolumn{2}{|c|}{$\frac{1983}{\text { Alcaldesas }}$}} & \multirow{2}{*}{\multicolumn{2}{|c|}{$\begin{array}{c}1991 \\
\text { Alcaldesas }\end{array}$}} & \multicolumn{4}{|c|}{1995} & \multicolumn{4}{|c|}{1999} & \multicolumn{4}{|c|}{2007} \\
\hline & & & & & \multicolumn{2}{|c|}{ Alcaldesas } & \multicolumn{2}{|c|}{ Regidoras } & \multicolumn{2}{|c|}{ Alcaldesas } & \multicolumn{2}{|c|}{ Regidoras } & \multicolumn{2}{|c|}{ Alcaldesas } & \multicolumn{2}{|c|}{ Regidoras } \\
\hline & $\mathrm{M}^{1}$ & $\%$ & $\mathrm{M}$ & $\%$ & $\mathrm{M}$ & $\%$ & $\mathrm{M}$ & $\%$ & M & $\%$ & $\mathrm{M}$ & $\%$ & $\mathrm{M}$ & $\%$ & $\mathrm{M}$ & $\%$ \\
\hline Andalucía & 16 & 2,1 & 14 & 1,8 & 30 & 3,9 & 1.520 & 19,3 & 60 & 7,8 & 2.210 & 25,9 & 123 & 16,0 & 3.378 & 37,80 \\
\hline Aragón & 13 & 1,8 & 36 & 4,9 & 50 & 6,9 & 464 & 13,4 & 69 & 9,5 & 700 & 16,6 & 98 & 13,4 & 979 & 23,12 \\
\hline Asturias & 3 & 3,8 & 3 & 3,8 & 3 & 3,8 & 194 & 21,2 & 5 & 6,4 & 260 & 26,7 & 11 & 14,1 & 355 & 37,45 \\
\hline Islas Baleares & 1 & 1,5 & 5 & 7,5 & 7 & 10,4 & 161 & 20,9 & 6 & 9,0 & 205 & 24,3 & 6 & 9,0 & 321 & 35,91 \\
\hline Islas Canarias & 1 & 1,1 & 2 & 2,3 & 2 & 2,3 & 215 & 18,3 & 4 & 4,6 & 309 & 24,3 & 11 & 12,6 & 522 & 38,27 \\
\hline Cantabria & 4 & 3,9 & 4 & 3,9 & 3 & 2,9 & 122 & 13,3 & 4 & 3,9 & 196 & 19,3 & 12 & 11,8 & 282 & 27,30 \\
\hline Castilla-León & 52 & 2,3 & 123 & 5,5 & 170 & 7,6 & 1.339 & 12,3 & 242 & 10,8 & 2.007 & 15,9 & 305 & 13,6 & 2.835 & 22,86 \\
\hline Castilla-La Mancha & 19 & 2,1 & 62 & 6,8 & 79 & 8,6 & 828 & 15,9 & 123 & 13,4 & 1.353 & 22,9 & 161 & 17,5 & 1.903 & 30,66 \\
\hline Cataluña & 15 & 1,6 & 34 & 3,6 & 44 & 4,7 & 1.101 & 15,3 & 66 & 7,0 & 1.528 & 18,4 & 120 & 12,7 & 2.585 & 29,05 \\
\hline Extremadura & 12 & 3,2 & 16 & 4,2 & 22 & 5,8 & 504 & 17,8 & 29 & 7,6 & 764 & 22,4 & 60 & 15,7 & 1.966 & 35,04 \\
\hline Galicia & 6 & 1,9 & 11 & 3,5 & 14 & 4,5 & 408 & 11,3 & 10 & 3,2 & 585 & 15,4 & 25 & 8,0 & 1.064 & 31,83 \\
\hline Madrid & 4 & 2,2 & 15 & 8,4 & 19 & 10,6 & 343 & 23,4 & 21 & 11,7 & 483 & 29,1 & 35 & 19,6 & 1.143 & 29,84 \\
\hline Murcia & 4 & 8,9 & 2 & 4,4 & 3 & 6,7 & 140 & 21,7 & 3 & 6,7 & 178 & 26,7 & 6 & 13,3 & 850 & 38,76 \\
\hline Navarra & 1 & 0,4 & 14 & 5,2 & 14 & 5,1 & 284 & 17,2 & 30 & 11,0 & 399 & 21,2 & 43 & 17,7 & 277 & 37,23 \\
\hline La Rioja & 5 & 2,9 & 10 & 5,7 & 14 & 8,0 & 504 & 22 & 14 & 8,0 & 181 & 19,9 & 18 & 10,3 & 895 & 35,03 \\
\hline Valencia & 3 & 0,6 & 19 & 3,5 & 33 & 6,1 & 136 & 16,8 & 63 & 11,6 & 1.451 & 27,0 & 95 & 17,5 & 260 & 26,89 \\
\hline País Vasco & 6 & 2,6 & 23 & 9,3 & 22 & 8,8 & 1.037 & 21,4 & 30 & 12,0 & 630 & 26,6 & 50 & 19,9 & 531 & 27,80 \\
\hline Estado español & 164 & 2,0 & 393 & 4,9 & 529 & 6,5 & 9.300 & 16,5 & 779 & 9,6 & 13.446 & 21,1 & 1.179 & 14,6 & 20.165 & 30,5 \\
\hline Total ambos sexos & \multicolumn{2}{|c|}{8.051} & \multicolumn{2}{|c|}{8.086} & \multicolumn{2}{|c|}{8.096} & \multicolumn{2}{|c|}{56.537} & \multicolumn{2}{|c|}{8.105} & \multicolumn{2}{|c|}{63.731} & \multicolumn{2}{|c|}{8.075} & \multicolumn{2}{|c|}{66.115} \\
\hline
\end{tabular}

1. M: Mujeres;

Fuente: Calculado por las autoras - autor en base a los datos de los parlamentos autonómicos.

Disponível em: $<$ http://www.inmujer.migualdad.es/mujer/... >, 2010.

\begin{tabular}{|c|c|c|c|c|c|c|c|}
\hline \multirow[t]{2}{*}{ Comarca } & \multicolumn{2}{|c|}{ Representantes políticos } & \multicolumn{2}{|c|}{ Técnicos y especialistas } & \multicolumn{3}{|c|}{$\begin{array}{l}\text { Representantes municipales: } \\
\text { regidoras y regidores }\end{array}$} \\
\hline & Mujeres & Hombres & Mujeres & Hombres & Mujeres & Hombres & Total \\
\hline Alta Ribagorça & 1 & 1 & 0 & 0 & 6 & 21 & 27 \\
\hline Alt Urgell & 4 & 4 & 1 & 1 & 26 & 100 & 126 \\
\hline Cerdanya & 2 & 2 & 0 & 1 & 28 & 80 & 108 \\
\hline Pallars Jussà & 2 & 2 & 0 & 1 & 20 & 77 & 97 \\
\hline Pallars Sobirà & 2 & 2 & 1 & 0 & 21 & 77 & 98 \\
\hline Vall d'Aran & 1 & 1 & 1 & 0 & 11 & 44 & 55 \\
\hline $\mathrm{APC}$ & 12 & 12 & 3 & 3 & 112 & 399 & 512 \\
\hline
\end{tabular}

Fuente: elaboración propia en base a las entrevistas realizadas el año 2011 y datos Corcoy (2009).

\section{El Alto Pirineo Catalán como área de estudio}

La región más montañosa de Cataluña es l'Alt Pirineu i Aran (APC, Figura 1), con un territorio de $5.686 \mathrm{~km} 2$ y $76.662 \mathrm{~h}$ el año 2010. El número de habitantes de la APC es el 1,02\% de Cataluña mientras que su superficie es el $17,8 \%$. La proporción de mujeres sobre el total de Cataluña ha sido siempre inferior a la de los hombres (Tabla 3). El proceso dominante en el siglo XX ha sido la emigración hacia las áreas urbanas del litoral catalán, emigrando más las mujeres que los hombres por la ya comentada figura del hereu y la falta de oportunidades laborales y personales. En su conjunto, la población de estas comarcas de montaña fue disminuyendo hasta un mínimo en el censo de 1991, y después se ha
Figura 1. Área de estudio: Alto Pirineo Catalán (APC) y las 6 comarcas de montaña

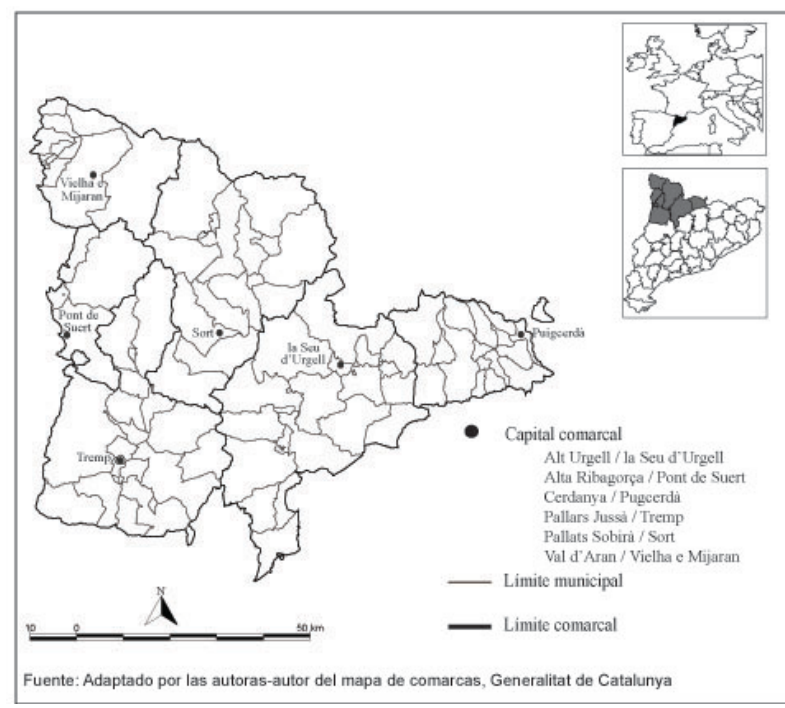

Antoni F. Tulla, Antonia Casellas, Marta Pallares-Blanch e Ana Vera 


\begin{tabular}{|c|c|c|c|c|c|c|c|c|c|}
\hline & \multicolumn{3}{|c|}{1975} & \multicolumn{3}{|c|}{1991} & \multicolumn{3}{|c|}{2010} \\
\hline Comarcas & Hombres & Mujeres & Total & Hombres & Mujeres & Total & Hombres & Mujeres & Total \\
\hline Alt Urgell & 9.562 & 9.226 & 18.788 & 9.667 & 9.343 & 19.01 & 11.126 & 10.879 & 22.005 \\
\hline Alta Ribagorça & 2.195 & 2.081 & 4.276 & 1.813 & 1.701 & 3.514 & 2.289 & 1.989 & 4.278 \\
\hline Cerdanya & 6.46 & 6.149 & 12.609 & 6.309 & 6.087 & 12.396 & 9.642 & 8.907 & 18.549 \\
\hline Pallars Jussà & 7.487 & 7.292 & 14.779 & 6.413 & 6.447 & 12.86 & 7.047 & 6.931 & 13.978 \\
\hline Pallars Sobirà & 3.221 & 2.894 & 6.115 & 2.814 & 2.604 & 5.418 & 4.034 & 3.612 & 7.646 \\
\hline Val d'Aran & 2.693 & 2.477 & 5.17 & 3.127 & 3.057 & 6.184 & 5.297 & 4.909 & 10.206 \\
\hline $\mathrm{APC}$ & 31.618 & 30.119 & 61.737 & 30.143 & 29.239 & 59.382 & 39.435 & 37.227 & 76.662 \\
\hline Catalunya & 2.763 .107 & 2.897 .265 & 5.660 .372 & 2.962 .942 & 3.096 .552 & 6.059 .494 & 3.724 .515 & 3.787 .866 & 7.512 .381 \\
\hline$\%$ APC $/$ Cat & 1,14 & 1,04 & 1,09 & 1,02 & 0,94 & 0,98 & 1,06 & 0,98 & 1,02 \\
\hline
\end{tabular}

Fuente: Idescat. Bases de datos de municipios (2011)

recuperado hasta un máximo en el 2010. En el año 2008, más del $15 \%$ de la población era inmigrada (SOLÉ, 2009)

a tasa de ocupación femenina ${ }^{2}$ en el APC es del $42,3 \%, 4$ puntos inferior a la tasa de ocupación media femenina de Cataluña (46,3\%) y algo superior a la tasa de ocupación media femenina de España, de un 41,6\% (datos de 2010). La tasa de ocupación masculina ${ }^{3}$ en el APC es del 52,4\%, 10 puntos superior a la de las mujeres y siendo también inferior a la media de Cataluña; 57,3\% y también inferior, aunque con menos diferencia de la media española, del 54,6\%. La tasa de actividad femenina ${ }^{4}(42,3 \%)$ es inferior a la masculina $(65,5 \%)$ en el APC como sucede también para Cataluña (55,5\% y 70,4\%) y España (52,3\% y 68,1\%) (datos de 2010) (IDESCAT, 2011). Esta situación se explicaría, en parte, por un mayor número de mujeres en economía sumergida.

La distribución del empleo por sectores en las distintas comarcas del APC se presenta en la Tabla 4.

\begin{tabular}{|l|r|r|r|r|}
\hline \multicolumn{5}{|c|}{ Tabla 4. Empleo* por sectores APC \& Cataluña (2009), \% } \\
\hline \multicolumn{1}{|c|}{ Comarca } & \multicolumn{1}{c|}{ Agrario } & Industrial & Construcción & \multicolumn{1}{c|}{ Servicios } \\
\hline Alt Urgell & 7,8 & 14,3 & 14,3 & 63,6 \\
\hline Alta Ribagorça & 5,0 & 5,5 & 15,9 & 73,5 \\
\hline Cerdanya & 5,0 & 4,3 & 19,4 & 71,2 \\
\hline Pallars Jussà & 13,0 & 6,8 & 15,1 & 65,0 \\
\hline Pallars Sobirà & 8,3 & 4,4 & 15,9 & 71,4 \\
\hline Vall d'Aran & 0,8 & 2,2 & 10,5 & 86,5 \\
\hline APC & 6,6 & 6,6 & 15,3 & 71,6 \\
\hline Cataluña & 1,2 & 16,6 & 8,9 & 73,3 \\
\hline
\end{tabular}

Fuente: elaborado por las autoras-autor en base al Observatori del Treball www.gencat.cat, 2009. *Población activa

\section{La relación de Género y Política en el Alto Pirineo Catalán (APC)}

Las políticas de género han avanzado durante los últimos veinte años, aunque de manera más efectiva en las áreas urbanas que en las rurales. La construcción de los espacios urbanos, incluso en las áreas rurales, se ha proyectado $\mathrm{y}$ realizado exclusivamente por hombres con criterios obsoletos sobre el papel de la mujer en la economía y la sociedad (ORTIZ, 2004). La incorporación de la perspectiva de género en los estudios de planificación pública aparece actualmente como uno de los retos que deben afrontar las áreas rurales. La participación de las mujeres en la toma de decisiones en las políticas públicas puede ser una oportunidad en la situación de la crisis actual debido a una mayor efectividad de su gestión (BENERÍA, 2010).

Es importante recordar el trabajo pionero de Leonore Davidoff et al (1976), citado por Jo Little y Ruth Panelli (2003), que identifica una ideología dominante en las sociedades rurales de occidente donde se posiciona a los hombres a la cabeza de una 'jerarquía natural' y a las mujeres, en su papel doméstico en la esfera reproductiva, como sostén del modo de vida y de la formación social representada por la familia, la comunidad o el pueblo. Estas condiciones han ido cambiando con la diversificación productiva y el papel emergente de la mujer en la toma de decisiones. Al estudiar la introducción de las actividades agroturísticas en la España rural, se ha podido identificar a las mujeres como agentes emprendedores que organizan y realizan la mayor parte de las actividades de este nuevo tipo de economía (GARCÍA-RAMÓN, 1995). Esta nueva situación ha favorecido la participación de la mujer en cargos electos de la administración local en Cataluña, 
aumentando del 4,8\% en 1979 al $29,6 \%$ en el 2007 y también en el APC, al pasar del 3,9\% al $21,9 \%$ en el mismo período (Tabla 6).

La doble discriminación de la mujer en términos de producción y de reproducción en el trabajo se acentúa en el APC por ser un área periférica donde hay menos opciones de empleo y la administración local tiene menor capacidad para proveer servicios a la comunidad (transporte, ocio, atención de enfermos, personas de edad y niños, etc.). Estas circunstancias convierten a las mujeres en 'proveedoras de cuidados' junto a su papel de 'pilar de la familia' y pieza clave en la transmisión del patrimonio cultural y social de las áreas rurales (PALLARÈS-BARBERÀ, 2003).

En las entrevistas realizadas hay una gran coincidencia entre la participación de las mujeres en puestos electos de la administración local y el hecho de que hayan sido requeridas por hombres que buscan completar listas electorales, por falta de candidatos o por garantizar 'una cuota de género'. Las mujeres que aceptan son, en general, mujeres con hijos ya mayores, mujeres sin hijos, o mujeres con hijos jóvenes pero con facilidades para la conciliación. Las mujeres, por lo general, repiten menos períodos legislativos (PL) que los hombres.

\section{La Participación de las Mujeres en la Administración Local}

La falta de una red urbana importante en el APC y la fuerte emigración, ha impulsado a los jóvenes $\mathrm{y}$ especialmente a las mujeres mejor preparadas a instalarse en las grandes ciudades de Cataluña, representando una pérdida de las personas más bien formadas que dificultan los procesos de innovación productiva y organizativa (PALLARÈS-BLANCH, 2009). La participación de la mujer en los consistorios municipales en las áreas urbanas del APC (Tabla 5) es menor que en el conjunto del APC $(21.9 \%)$ o de

\begin{tabular}{|c|c|c|c|c|c|c|c|}
\hline $\begin{array}{c}\text { Comarca } \\
\text { Ciudad o pueblo } \\
\text { cabecera del valle }\end{array}$ & $\begin{array}{c}\% \\
\text { regidores } \\
1 \text { er PL } \\
1979-83 \\
\end{array}$ & $\begin{array}{c}\% \\
\text { regidores } \\
8^{\circ} \mathrm{PL} \\
2007-11 \\
\end{array}$ & $\begin{array}{l}\text { Población } \\
\text { total }\end{array}$ & $\begin{array}{l}\text { \% de la } \\
\text { región } \\
\text { estudiada }\end{array}$ & $\begin{array}{l}\% \text { de la } \\
\text { comarca }\end{array}$ & $\begin{array}{l}\text { Superficie } \\
\left(\mathrm{km}^{2}\right)\end{array}$ & $\begin{array}{c}\text { Densidad } \\
\text { de } \\
\text { Población } \\
\left(\mathrm{h} / \mathrm{km}^{2}\right) \\
\end{array}$ \\
\hline Alt Urgell & 0,8 & 20,6 & 21.942 & 29 & 100 & 1.448 & 15 \\
\hline la Seu d'Urgell & 7,7 & 36,8 & 12.986 & 17 & 59 & 15 & 841 \\
\hline Oliana & 0,0 & 33,3 & 1.949 & 3 & 9 & 32 & 60 \\
\hline Organyà & 0,0 & 28,6 & 946 & 1 & 4 & 13 & 76 \\
\hline Alta Ribagorça & 4,0 & 22,2 & 4.332 & 6 & 100 & 427 & 10 \\
\hline el Pont de Suert & 0,0 & 36,4 & 2.553 & 3 & 59 & 148 & 17 \\
\hline Cerdanya & 2,8 & 25,9 & 18.658 & 24 & 100 & 547 & 34 \\
\hline Puigcerdà & 7,7 & 38,5 & 9.365 & 12 & 50 & 19 & 495 \\
\hline Alp & 0,0 & 22,2 & 1.686 & 2 & 9 & 44 & 38 \\
\hline Bellver & 0,0 & 44,4 & 2.132 & 3 & 11 & 98 & 22 \\
\hline Pallars Jussà & 5,2 & 20,6 & 13.715 & 18 & 100 & 1.343 & 10 \\
\hline Tremp & 23,1 & 38,5 & 6.190 & 8 & 45 & 303 & 20 \\
\hline Pobla de Segur & 0,0 & 45,5 & 3.151 & 4 & 23 & 33 & 96 \\
\hline $\begin{array}{r}\text { Isona i Conca } \\
\text { Dellà }\end{array}$ & 11,1 & 0,0 & 1.151 & 2 & 8 & 139 & 8 \\
\hline Pallars Sobirà & 2,1 & 21,4 & 7.446 & 10 & 100 & 1.378 & 5 \\
\hline Sort & 0,0 & 27,3 & 2.373 & 3 & 32 & 105 & 23 \\
\hline Val d'Aran & 13,6 & 20,0 & 10.194 & 13 & 100 & 634 & 16 \\
\hline Vielha & 9,1 & 30,8 & 5.633 & 7 & 55 & 212 & 27 \\
\hline $\begin{array}{r}\text { Salardú (Naüt } \\
\text { Aran) }\end{array}$ & 11,1 & 11,1 & 1.731 & 2 & 17 & 256 & 7 \\
\hline Bossost & 14,3 & 22,2 & 1.202 & 2 & 12 & 28 & 43 \\
\hline $\mathrm{APC}$ & 3,9 & 21,9 & 76.287 & 100 & 100 & 5.776 & 13 \\
\hline $\begin{array}{r}\text { Municipios } \\
\text { urbanos } \\
\end{array}$ & 6,2 & 30,9 & 53.048 & 69.5 & - & 1.445 & 38 \\
\hline Total Cataluña & 4,8 & 29,6 & 7.364 .078 & 100 & & 32.107 & 225 \\
\hline
\end{tabular}

Fuente: Idescat (2009) y Corcoy, ICPS (2007) 
Cataluña (29.6\%). Situación que es más extrema si sólo consideramos las mujeres en los municipios de las áreas rurales del Pirineo (18.1\%) (Tabla 6).

En los resultados provisionales de las elecciones municipales de 2011, ha aumentado hasta el 26,2\% la participación de las mujeres en el conjunto del APC inscriben fuera de las competencias que manejan los mayores presupuestos, lo cual demuestra que la sociedad rural del APC sólo les concede 'aparecer' pero no 'gobernar' (Tabla 7).

Esta situación de menor participación de la mujer en la política pública puede explicarse por tres

\begin{tabular}{|c|c|c|c|c|c|c|c|c|c|c|c|c|c|}
\hline & . & 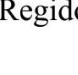 & 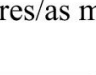 & 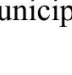 & 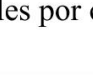 & $(19$ & 9-2011 & 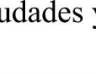 & 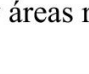 & 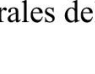 & 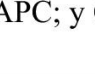 & 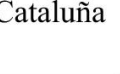 & \\
\hline \multirow[b]{2}{*}{ Comarcas } & \multicolumn{3}{|c|}{ 1er $\mathrm{PL}^{1} 1979-83$} & \multicolumn{3}{|c|}{$4^{\circ} \mathrm{PL} 1991-95$} & \multicolumn{3}{|c|}{$7^{\circ}$ PL 2003-07 } & \multicolumn{3}{|c|}{$8^{\circ}$ PL 2007-11 } & \multirow{2}{*}{$\begin{array}{c}\text { Indice }^{2} \\
\text { variación } \\
\text { mujeres } \\
\text { 2011/1979 }\end{array}$} \\
\hline & $\begin{array}{l}\mathrm{M} \\
\mathrm{N}\end{array}$ & $\begin{array}{l}\mathrm{M} \\
\%\end{array}$ & $\begin{array}{c}\text { Total } \\
\mathrm{N}\end{array}$ & $\mathrm{MN}$ & M \% & $\begin{array}{c}\text { Total } \\
\mathrm{N}\end{array}$ & $\begin{array}{l}\mathrm{M} \\
\mathrm{N}\end{array}$ & $\mathrm{M} \%$ & $\begin{array}{c}\text { Total } \\
\mathrm{N}\end{array}$ & $\begin{array}{l}\mathrm{M} \\
\mathrm{N}\end{array}$ & M \% & Total N & \\
\hline Alt Urgell & 1 & 0,8 & 131 & 8 & 6,4 & 125 & 21 & 17,5 & 120 & 26 & 20,6 & 126 & 96,15 \\
\hline $\begin{array}{l}\text { Alta } \\
\text { Ribagorça }\end{array}$ & 1 & 4,0 & 25 & 5 & 20,0 & 25 & 6 & 24,0 & 25 & 6 & 22,2 & 27 & 83,33 \\
\hline Cerdanya & 3 & 2,8 & 107 & 6 & 5,9 & 102 & 17 & 15,6 & 109 & 28 & 25,9 & 108 & 89,29 \\
\hline $\begin{array}{l}\text { Pallars } \\
\text { Jussà }\end{array}$ & 5 & 5,2 & 97 & 10 & 10,2 & 98 & 17 & 17,3 & 98 & 20 & 20,6 & 97 & 75,00 \\
\hline $\begin{array}{l}\text { Pallars } \\
\text { Sobirà }\end{array}$ & 2 & 2,1 & 97 & 11 & 12,4 & 89 & 21 & 23,1 & 91 & 21 & 21,4 & 98 & 90,48 \\
\hline Val d'Aran & 8 & 13,6 & 59 & 7 & 13,7 & 51 & 7 & 13,7 & 51 & 11 & 20,0 & 55 & 27,27 \\
\hline Total APC & 20 & 3,9 & 516 & 47 & 9,6 & 490 & 89 & 18,0 & 494 & 112 & 21,9 & 511 & 82,14 \\
\hline $\begin{array}{l}\text { Ciudades } \\
\text { ACP }\end{array}$ & 9 & 6,2 & 146 & 18 & 12,2 & 148 & 29 & 20,1 & 144 & 47 & 30,9 & 152 & 80,85 \\
\hline $\begin{array}{l}\text { Sólo área } \\
\text { rural }\end{array}$ & 11 & 3,0 & 370 & 29 & 8,5 & 342 & 60 & 17,1 & 350 & 65 & 18,1 & 359 & 83,08 \\
\hline Cataluña & 393 & 4,8 & 8.262 & 919 & 11,0 & 8.321 & 2.014 & 23,2 & 8.682 & 2.651 & 29,6 & 8.953 & 85,18 \\
\hline
\end{tabular}

${ }^{1}$ LP: Período Legislativo, M: Mujeres

${ }^{2}$ [(mujeres N 2007 - mujeres N 1979) / mujeres N 2007] x 100

Las áreas rurales son los municipios de menos de 750 habitantes y las ciudades los de más de 750 h. (con 9 o más regidurías municipales)

Fuente: "Mujeres y hombres en las instituciones democráticas de Cataluña," una base de datos compilada por Marta Corcoy Rius y Patricia Laura Gómez, Instituto de Ciencias Sociales y Políticas (ICPS), 2007.

mientras que en el conjunto de Cataluña ha superado el $35 \%$ (Tabla 6).

El número de alcaldesas, sin embargo es muy inferior al de regidoras. En la tabla 7 podemos comprobar que se ha pasado de un 2,6\% de mujeres el año 1979 en el APC a un 6,5\% en el 2007. En el conjunto de Cataluña se ha pasado del $2,2 \%$ al $13,0 \%$ en el mismo período. En las elecciones municipales del 2011, el número de alcaldesas en el APC ha aumentado hasta un $9,1 \%$ y en Cataluña se ha alcanzado el $14,1 \%$. Es evidente que hay un mayor aumento de participación de las mujeres como regidoras municipales en el APC $(26,2 \%)$ pero no llegan al $10 \%$ de alcaldesas y sus responsabilidades se motivos. Primero, por la prevalencia de la figura del hereu que ya hemos comentado, que determina que las estructuras de organización social y política funcionen con un código de valores más patriarcal que en las urbanas (BINIMELIS, 2008). Segundo, las mujeres del mundo rural viven una doble invisibilidad. Por un lado por la falta de reconocimiento explícito del trabajo realizado, dentro y fuera de la familia, y de las funciones que lleva a cabo en la comunidad y, por otro lado, por la posición que ocupan en una sociedad de tradición agraria donde los hombres han sido los únicos actores visibles de una rápida modernización (BAYLINA ; GARCIA-RAMON, 2004), hecho que se añade al hecho de ser ciudadanas de áreas rurales. 


\begin{tabular}{|c|c|c|c|c|c|c|c|c|c|c|c|c|c|}
\hline \multirow[b]{2}{*}{ Comarcas } & \multicolumn{3}{|c|}{ 1er PL ${ }^{1} 1979-83$} & \multicolumn{3}{|c|}{$4^{\circ}$ PL 1991-95 } & \multicolumn{3}{|c|}{$7^{\circ}$ PL 2003-07 } & \multicolumn{3}{|c|}{$8 \mathrm{t}^{\circ}$ PL 2007-11 } & \multirow{2}{*}{$\begin{array}{c}1979-07 \\
\% \mathrm{M}\end{array}$} \\
\hline & $\mathrm{M}^{2}$ & $\begin{array}{l}\mathrm{M} \\
\% \\
\end{array}$ & Total & $\mathrm{M}^{2}$ & \begin{tabular}{l|l|}
$\mathrm{M}$ \\
$\%$
\end{tabular} & Total & $\mathrm{M}^{2}$ & $\begin{array}{l}\mathrm{M} \\
\% \\
\end{array}$ & Total & $\mathrm{M}^{2}$ & $\begin{array}{l}\mathrm{M} \\
\% \\
\end{array}$ & Total & \\
\hline Alt Urgell & 0 & 0,0 & 19 & 0 & 0,0 & 19 & 0 & $\% \mathrm{M}$ & 19 & 2 & 10,5 & 19 & 10,5 \\
\hline $\begin{array}{l}\text { Alta } \\
\text { Ribagorça }\end{array}$ & 0 & 0,0 & 3 & 0 & 0,0 & 3 & 0 & 0,0 & 3 & 0 & 0,0 & 3 & 0,0 \\
\hline Cerdanya & 1 & 6,3 & 16 & 1 & 5,9 & 17 & 1 & 0,0 & 17 & 1 & 5,9 & 17 & $-0,4$ \\
\hline Pallars Jussà & 0 & 0,0 & 14 & 0 & 0,0 & 14 & 1 & 5,9 & 14 & 1 & 7,1 & 14 & 7,1 \\
\hline Pallars Sobirà & 0 & 0,0 & 15 & 0 & 0,0 & 15 & 2 & 7,1 & 15 & 1 & 6,7 & 15 & 6,7 \\
\hline Val d'Aran & 1 & 11,1 & 9 & 1 & 11,1 & 9 & 0 & 13,3 & 9 & 0 & 0,0 & 9 & $-11,1$ \\
\hline APC & 2 & 2,6 & 76 & 2 & 2,6 & 77 & 4 & 0,0 & 77 & 5 & 6,5 & 77 & 3,9 \\
\hline Cataluña & 21 & 2,2 & 934 & 30 & 3,2 & 941 & 95 & 5,2 & 945 & 124 & 13,0 & 955 & 10,7 \\
\hline
\end{tabular}

${ }^{1}$ PL: Período Legislativo; ${ }^{2} \mathrm{M}$ : Mujeres

Fuente: "Mujeres y hombres en las instituciones democráticas de Cataluña," una base de datos compilada por Marta Corcoy Rius y Patricia Laura Gómez, Instituto de Ciencias Sociales y Políticas (ICPS), 2007.

En las áreas rurales del APC esta situación ha cambiado poco aunque haya aumentado la igualdad de oportunidades en términos legales. La introducción masiva de las mujeres en el mercado laboral se produce más tarde que en las áreas urbanas, principalmente por la escasa oferta de trabajos para ellas y por existir mayores dificultades para la conciliación laboral, familiar $\mathrm{y}$ personal. Estas circunstancias se tienen en cuenta, cada vez más, en la agenda política y son muy relevantes a nivel local por las necesidades de guarderías, de atención a la gente mayor y el cuidado de los dependientes en general. Muchos ayuntamientos y consejos comarcales se han visto obligados a implementar estos servicios y ha sido entonces cuando se ha considerado importante incorporar mujeres en los cargos electos municipales. En las entrevistas realizadas, hay una valoración por parte de los hombres entrevistados, sobre la eficacia de las mujeres en la gestión de muchos servicios, como por ejemplo la provisión y potabilización de aguas, habiéndose planteado de forma más racional e higiénica por parte de las mujeres. También es importante añadir que cada vez hay más mujeres con responsabilidades técnicas (arquitectas, médicas, veterinarias, archiveras, etc.) o de gestión (secretaria de ayuntamiento, gerencia de las instituciones locales, etc.).

\section{El Desarrollo Local en el Alto Pirineo Catalán}

Existen diversas opciones de desarrollo en esta área de montaña. En primer lugar, la adaptación de las actividades tradicionales agrarias obteniendo productos artesanales de calidad (quesos de oveja o de cabra, productos de madera, etc.) o bien logrando un rendimiento económico competitivo en el mercado (fábricas de transformación de leche de vaca o mataderos de carne ecológica, etc.) debido al valor añadido del proceso. En segundo lugar, promoviendo actividades turísticas, algunas orientadas al turismo masivo (segundas residencias, estaciones de esquí alpino, etc.) y que es consumidor de suelo de valor agrario, y otras orientadas al turismo agrario, que se beneficia de la proximidad a los espacios naturales protegidos. En tercer lugar, la substitución de industrias obsoletas por el proceso de globalización (textil, minería, etc.) por servicios y otro tipo de industrias que utilizan las nuevas tecnologías (textil deportivo, talleres profesionales, etc.). En cuarto lugar, la extensión de los servicios personales (educación, sanidad, etc.) y de servicios culturales (archivos, museos, oficinas de patrimonio, etc.). Finalmente, en quinto lugar, la existencia de algunas cooperativas que favorecen la obtención de mejores precios de los productos en el mercado (TULLA, 2009).

La participación de la mujer en el desarrollo local del APC se ha llevado a cabo de dos formas distintas. En primer lugar, impulsando nuevas actividades como el turismo rural (GARCIA-RAMON, 1995) o la elaboración de productos artesanos, agroalimentarios o no, y en segundo lugar desde la administración local y autonómica, así como desde asociaciones de diverso tipo (PALLARES-BARBERA, 2003; 2005). En las entrevistas realizadas hemos comprobado que algunas de las mujeres que han optado a cargos electos en los ayuntamientos han sido también emprendedoras de actividades de valor añadido en el APC. Los hombres entrevistados reconocen que las mujeres han sido más sensibles en la promoción de nuevas actividades artesanas relacionadas con el turismo. La 
transformación del espacio rural en esta área de montaña ha combinado la producción familiar con la comercialización a través de mercados, ferias y la promoción de jornadas que han favorecido la creación de una marca distintiva de algunos productos como quesos, carne de vacuno etc. (TULLA, 2003).

\section{La Responsabilidad de las Mujeres en la Gestión Municipal}

En la distribución de cargos municipales entre los regidores en el gobierno de los municipios (Tabla 8) se puede observar, aún en el PL 2007-11, que en el APC un $71,4 \%$ de las mujeres elegidas no tienen responsabilidades específicas. En cambio, si comparamos con toda Cataluña el $57,5 \%$ de mujeres tienen cargo asignado.

Las responsabilidades de las regidoras se centran en los servicios sociales, tanto en Cataluña como en el
APC y, en menor grado, en la administración, y en territorio y medio ambiente. Sin embargo, las entrevistas demuestran dos hechos: primero, que en los municipios del APC, con excepción de alguna ciudad, hay poca especialización de los miembros del gobierno municipal y segundo, que las responsabilidades van cambiando continuamente y muchas veces son compartidas, apareciendo en primer lugar el regidor hombre.

\section{¿Hay una Menor Incidencia de las Mujeres en la Gobernanza de las Áreas Rurales?}

En esta investigación, iniciada con la revisión bibliográfica sobre "mujer y política" y "mujer y desarrollo económico", reforzada con el análisis cuantitativo de los datos de los cargos electos municipales desde la primera LP (1979-83) a la octava LP (2007-11), y completada con el análisis cualitativo

Tabla 8. Responsabilidad de las mujeres en los consejos municipales del APC y en Cataluña (PL 2007-11) ${ }^{1}$

\begin{tabular}{|c|c|c|c|c|c|c|c|}
\hline \multirow{2}{*}{$\begin{array}{c}\text { Tipo de responsabilidad en el } \\
\text { Consejo Municipal }\end{array}$} & \multicolumn{3}{|c|}{ APC } & \multicolumn{3}{|c|}{ Cataluña } & \multirow{2}{*}{$\begin{array}{c}\% \text { diferencia } \\
\text { de mujeres } \\
\text { entre el } \\
\text { APC/Cat }{ }^{2}\end{array}$} \\
\hline & $\begin{array}{l}\text { Mujeres } \\
\qquad \mathrm{N}\end{array}$ & $\begin{array}{l}\text { Mujeres } \\
\%\end{array}$ & Total N & $\begin{array}{l}\text { Mujeres } \\
\mathrm{N}\end{array}$ & $\begin{array}{l}\text { Mujeres } \\
\%\end{array}$ & $\begin{array}{l}\text { Total } \\
\mathrm{N}\end{array}$ & \\
\hline $\begin{array}{l}\text { Administración y Agencia } \\
\text { tributaria }\end{array}$ & 10 & 13,3 & 75 & 146 & 15,0 & 975 & $-11,33$ \\
\hline $\begin{array}{l}\text { Mantenimiento. Infraestructuras } \\
\text { y servicios municipales }\end{array}$ & 1 & 12,5 & 8 & 29 & 11,9 & 244 & 5,04 \\
\hline $\begin{array}{l}\text { Participación ciudadana. Ayuda } \\
\text { ciudadana. Cooperación e } \\
\text { Igualdad }\end{array}$ & 0 & 0,0 & 4 & 38 & 31,4 & 121 & $-100,00$ \\
\hline Servicios sociales & 16 & 29,1 & 55 & 724 & 43,0 & 1.682 & $-32,33$ \\
\hline Territorio y Medio Ambiente & 2 & 4,9 & 41 & 101 & 11,3 & 891 & $-56,4$ \\
\hline $\begin{array}{l}\text { Comunicaciones y Nuevas } \\
\text { tecnologías }\end{array}$ & 0 & - & 0 & 5 & 25,0 & 20 & $-100,00$ \\
\hline $\begin{array}{l}\text { Desarrollo y actividades } \\
\text { económicas }\end{array}$ & 3 & 17,6 & 17 & 83 & 20,0 & 415 & $-12,00$ \\
\hline Sin responsabilidades & 80 & 25,7 & 311 & 1.525 & 33,1 & 4.605 & $-22,6$ \\
\hline$\%$ sin responsabilidades/ Total & - & 71,4 & 60,9 & - & 57,5 & 51,4 & $-10,28$ \\
\hline$\%$ con responsabilidades/ Total & - & 28,6 & 39,1 & - & 42,5 & 48,6 & $-32,70$ \\
\hline Total & 112 & 21,9 & 511 & 2.651 & 29,6 & 8.953 & $-26,01$ \\
\hline
\end{tabular}

${ }^{1} \mathrm{PL}$ : Periodo legislativo

${ }^{2}$ [(mujeres \% APC - mujeres \% Cataluña) : mujeres \% Cataluña $x 100$

Fuente: Base de datos compilada por Marta Corcoy y Patricia Laura , (ICPS), 2007 
de las entrevistas realizadas, hemos llegado a diversas conclusiones: (a) existe un progreso lento de mayor participación de las mujeres en los cargos electos de la administración local; (b) este progreso aún es más lento en las áreas rurales del APC por la persistencia de diversas formas de patriarcado, económico, social y político; (c) la transformación socioeconómica ha favorecido la entrada laboral de la mujer en el mercado de trabajo y su participación en la política local; (d) no obstante aún es testimonial el número de mujeres que ocupan el cargo de alcaldesa; y (e) la participación activa de las mujeres en distintas experiencias de desarrollo local ha puesto de relieve su capacidad y efectividad en el liderazgo e innovación económica, lo cual ha fortalecido sus posibilidades de acceder a la gobernanza en los municipios rurales.

La participación de las mujeres en política viene determinada por diversos factores de oferta y demanda. Los primeros se refieren al número de candidatas que se ve afectado por cuestiones como pueden ser un menor interés por afiliarse a los partidos, una percepción de baja eficacia de la política, por un capital político disponible más bajo (preparación y background ocupacional), así como las dificultades de conciliación de la vida privada (familiar y personal), la laboral y la, relacionada con la participación pública y en asociaciones. En cuanto a los factores de demanda, la mujer debe hacer frente a una serie de factores relativos al acceso a los cargos públicos. La primera barrera es la cultura política dominante en los centros de poder, de más o menos explícita mentalidad sexista y supedita a los intereses del cacique de claro contenido patriarcal. En los partidos políticos, la definición de los méritos y cualidades para acceder a un cargo se orientan a partir de las características masculinas y las mujeres están sujetas a un examen de competencia continuado (NORRIS, 1997).

Estas circunstancias se ven reforzadas con una cultura organizativa de raíces patriarcales en las áreas de montaña que se traduce en la creación de redes informales de confianza y poder estrictamente masculinas, que se han consolidado a través de los años por la posición del cacique local o por la participación en actividades lúdicas como la caza. Por último, los horarios de la vida política no acostumbran a tener en cuenta la coincidencia con horas en que las mujeres acostumbran a estar más ocupadas por la atención de los hijos o de personas dependientes. Se puede verificar en nuestro estudio, la existencia del denominado 'techo de cristal' que impide a las mujeres alcanzar las posiciones más altas en su campo de actividad (VALCARCEL, 1997).
La crisis de las actividades económicas en el ámbito rural ha comportado diversos tipos de estrategias en Europa desde hace casi 50 años. Se plantea de forma general mejorar la producción agraria con una especialización competitiva o con la elaboración de productos artesanos de calidad (TULLA, 1994; 2009).

Las entrevistas de nuestra investigación nos han permitido demostrar que en buena parte de las nuevas actividades en el ámbito rural participan mujeres, con mayor o menor responsabilidad. En varios casos, las mujeres con cargos públicos en los ayuntamientos han sido impulsoras de actividades de desarrollo local, al ser a menudo ellas mismas emprendedoras en el ámbito del turismo rural y la artesanía de diversos tipos. Esta realidad no aparece de forma clara en las responsabilidades de gestión municipal (Tabla 8) lo cual pone en evidencia esta 'actividad invisible' de la mujer política. La revalorización de los espacios naturales en el APC ha facilitado este valor añadido en el turismo familiar (PRADOS, 2009) donde la mujer ocupa un lugar básico para su éxito. La crisis industrial en las áreas rurales ha implicado la necesidad de nuevas actividades para compensar el desempleo de mujeres en la industria textil y de pequeños electrodomésticos. También, algunas de las regidoras entrevistadas han sido activas en esta búsqueda de alternativas, no sólo en las distintas formas de turismo sino también en la incorporación de nuevas tecnologías en otros tipos de industrias y servicios para substituir el empleo (PALLARESBARBERA, 2003; VERA, 2003).

* Realizado con ayuda de los proyectos: RyC2008-02456, MICIIN CSO2009-08271 y ICDs2010 U $-9 / 10$

${ }^{1}$ El heredero en las áreas rurales de Cataluña es por tradición el varón primogénito, o el varón mayor. En caso de no haber varones es la mujer primogénita pubilla.

2 Proporción de mujeres trabajando/mujeres entre 16 y 65 años.

${ }^{3}$ Proporción de hombres trabajando/hombres entre 16 y 65 años.

4 Proporción de mujeres que forman parte de la población activa (trabajen o no)/ mujeres entre 16 y 65 años. De forma similar para los hombres. 


\section{Referências}

BAYLINA, Mireia; GARCIA-RAMON, Maria Dolors. Rural Gender Studies in Spain between 1975 and the Present. In: GOVERDE, Henri; DE HAAN, Henk; BAYLINA, Mireia (Orgs.) Power and gender in european rural development. Aldershot (England): Ashgate Publishing, 2004

BAYLINA, Mireia; BOCK, Bettina. Introduction to Part II: Gender and Rural Development in Academic Discourse. In: GOVERDE, Henri; DE HAAN, Henk; BAYLINA, Mireia (Orgs). Power and gender in european rural development. Aldershot (England): Ashgate Publishing, 2004.

BENERÍA, Lourdes; SARASÚA, Carmen ¿A quién afecta el recorte del gasto?. Diario El País, Disponível em: < http://www.elpais.com/articulo/opinion/...> Acesso em 28 out 2010.

BINIMELIS, Rosa; BOSCH, Mariona; HERRERO, Amaranta. A sol i serena: Dones, món rural i pagesia. Barcelona. Institut Català de les Dones, 2008.

CAMPILlO, Xavier; GANYET, Ramon; SANCLIMENS, Xavier. La població: evolució recent, situació actual i prospectiva per a l'any 2000. MAB-6 Alt Pirineu, n. 8, La Seu d’Urgell, 1987.

CASELLAS, Antonia; PALLARÈS-BLANCH, Marta; TULLA, Antoni F. Women's political participation in parliamentary democracy in rural Catalonia (1970's 2000's). Analele Universității de Vest din Timişoara, GEOGRAFIE, v. 19, p. 11.26, 2009. Disponível em: < http://www.geografie.uvt.ro/en/research/...>

CLOUT, Huge D. Geografía rural, (edición española 1976) Vilassar de Mar: Ed. Oikos Tau, 1972.

CORCOY, Marta; GÓMEZ, Patricia Laura. Elaboración de la Base de datos Dones i homes protagonistes a les institucions democràtiques catalanes 1977-2008. Institut de Ciències Polítiques i Socials, Barcelona, 2009.

DAVIDOFF, Leonore; L'ESPERANCE, Joan; NEWBY, Howard. Landscape with figures: home and community in English Society. En: MITCHELL, Juliet; OAKLEY, Ann. (Orgs) The rights and wrongs of women. Harmondsworth: Penguin, p. 379.399, 1976.
DUNCAN, Simon. The diverse worlds of European Patriarchy. En: GARCÍA-RAMON, Maria Dolors; MONK, Janice (Orgs) Women of the european union. The politics of work and daily life. London: Routledge, p.74.110, 1996

GARCIA-RAMON, Maria Dolors; CÀNOVES, Gemma; VALDOVINOS, Núria. Farm tourism, gender and the environment in Spain. Annals of Tourism Research, n. 22 - 2, p. 267-282, 1995.

GENERALITAT DE CATALUNYA. Observatori del treball. Barcelona, 2009.

GUIRADO, Carles; TULLA, Antoni F. Entre l'abandonament i l'ús intensiu del territorio? Sistema d'assentaments i gestió del territorio en espais de muntanya. El cas de l'Alt Pirineu català. Documents d'Anàlisi Geogràfica, v. 56, n. 3, p. 607-623, 2010.

IDESCAT. Estadística bàsica de Catalunya. Barcelona, 2011. Disponível em: < http://www.idescat.cat/ >

INSTITUTO DE LA MUJER. Ministerio de Sanidad, servicios sociales e igualdad. Estadísticas. 2012, Disponível em: < http://www.inmujer.es/ss/... >

LITTLE, Jo; PANELLI, Ruth. Gender Research in Rural Geography. Gender, Place and Culture, v. 10, n. 3. p. 281-289, September 2003.

NORRIS, Pippa. Passages to power. Legislative recruitment in advanced democracies. Cambridge: Cambridge University Press, 1997.

OLIVER, Josep. Anuari Econòmic Comarcal 2011. Estimació Del PIB comarcal 2010. Barcelona: Catalunya Caixa, 2011.

ORTIZ, Anna. Ús i apropiación de la Via Júlia i la rambla del Raval de Barcelona des d'una perspectiva de gènere. Documents d'Anàlisi Geogràfica, v. 44, p. 89-108, 2004.

PALLARÈS-BARBERÀ, Montse; TULLA, Antoni F.; PALLARÈS-BLANCH, Marta. Capital social i treball de les dones als pirineus. El cas de l'Alt Urgell. Barcelona : Institut Català de les Dones, 2003.

PALLARÈS-BARBERÀ, Montse; CASELLAS, Antònia; DOT, Esteve; PALLARÈS-BLANCH, Marta. 
Xarxes, Capital Social i treballs de les Dones als Pirineus. Barcelona, Institut Català de les Dones, 2005 (informe no publicado).

PALLARÈS-BLANCH, Marta. The benefits of Nature Reserve Areas in local development: An opportunity to develop a sustainable strategy in peripheral areas. En: Prados, Maria José (Orgs) Naturbanization: New identities and processes for rural-natural areas. London: Taylor \& Francis Group, 2009.

PRADOS, M. José. (Ed) Naturbanization: New identities and processes for rural - natural areas. London: Taylor \& Francis Group, 2009.

REDCLIFT, Nanneke; WHATMORE, Sarah. Hosehold, Consumption and Livelihood: Ideologies and Issues in Rural Research. En: MARSDEN, Terry; LOWE, Philip; WHATMORE, Sarah (Orgs) Rural Restructuring. Global Processes and their Responses. London: David Fulton Publishers. p. 182197,1990

SLEE, Bill. Alternative Farm Enterprises. Ipswich: Farming Press, 1987.

SOLÉ, Aimada. La incidència de la immigració estrangera a l'Alt Pirineu i Aran. Informe pel DPTOP de la Generalitat de Catalunya. Barcelona: Departament de Geografia de la UAB, 2009. (informe no publicado)

TULLA, Antoni F. Women and family farms in Catalonia. Iberian Studies, n. 20 (1 \& 2), p. 62-80, 1991.

TULLA, Antoni F. Procès de transformación agrària en àrees rurals de muntanya. Barcelona: Institut Cartogràfic de Catalunya, 1994.

TULLA, Antoni F. Funciones y procesos característicos del espacio rural. En: GARCIARAMON, Maria Dolors;

TULLA, Antoni F.; VALDOVINOS, Núria, Geografía Rural, Madrid: Síntesis, p. 53-89, 1995.

TULLA, Antoni F; SORIANO, J. Manuel; PALLARÈS, Montse; VERA, Ana . La transformació del model agrari en árees de muntanya. ESPAIS, 49, p. 82-97, 2003.
TULLA, Antoni F.; PALLARÈS-BARBERÀ, Montse; VERA, Ana. Naturbanization and local development in the mountain areas of the Catalan Pyrenees. En: PRADOS, M. José (Orgs) Naturbanization: New identities and processes for rural - natural areas. London: Taylor; Francis Group, 2009.

VALCARCEL, Amelia. La política de las mujeres. Madrid: Cátedra, 1997.

VERA, Ana; BADIA, Anna; PALLARES-BARBERA, Montse. La adopción de Internet en la red de empresas y la percepción de la nueva economía en comarcas semiremotas de tradición industrial de Catalunya. Boletín de la Asociación de Geógrafos Españoles, 36 , p. 129-147, 2003.

Recebido em: 25 de novembro de 2011. Aceito em: 20 de janeiro de 2012. 